ASTL.CLS 05.10.08

\title{
Fast optical variability of SS 433
}

\author{
R. A. Burenin ${ }^{* 1}$, M. G. Revnivtsev ${ }^{1,2}$, I. M. Khamitov³ , I. F. Bikmaev ${ }^{4,5}$, A. S. Nosov ${ }^{1}$, \\ M. N. Pavlinsky ${ }^{1}$, R. A. Sunyaev ${ }^{1,6}$ \\ ${ }^{1}$ Space Research Institute (IKI), Moscow, Russia \\ ${ }^{2}$ Excellence Cluster Universe, Technische Universität München, Germany \\ ${ }^{3}$ TUBITAK National Observatory, Antalya, Turkey \\ ${ }^{4}$ Kazan (Volga Region) State University, Kazan, Russia \\ ${ }^{5}$ Academy of Sciences of Tatarstan, Kazan, Russia \\ ${ }^{6}$ Max-Planck-Institut für Astrophysik, Garching, Germany
}

Received April 13, 2010

\begin{abstract}
We study the optical variability of the peculiar Galactic source SS 433 using the observations made with the Russian Turkish 1.5-m telescope (RTT150). A simple technique which allows to obtain high-quality photometric measurements with $0.3-1 \mathrm{~s}$ time resolution using ordinary CCD is described in detail. Using the test observations of nonvariable stars, we show that the atmospheric turbulence introduces no significant distortions into the measured light curves. Therefore, the data obtained in this way are well suited for studying the aperiodic variability of various objects.

The large amount of SS 433 optical light curve measurements obtained in this way allowed us to obtain the power spectra of its flux variability with a record sensitivity up to frequencies of $\sim 0.5 \mathrm{~Hz}$ and to detect its break at frequency $\approx$ $2.4 \times 10^{-3} \mathrm{~Hz}$. We suggest that this break in the power spectrum results from the smoothing of the optical flux variability due to a finite size of the emitting region. Based on our measurement of the break frequency in the power spectrum, we estimated the size of the accretion-disk photosphere as $2 \times 10^{12} \mathrm{~cm}$. We show that the amplitude of the variability in SS 433 decreases sharply during accretion-disk eclipses, but it does not disappear completely. This suggests that the size of the variable optical emission source is comparable to that of the normal star whose size is therefore $R_{O} \approx 2 \times 10^{12} \mathrm{~cm}$ $\approx 30 R_{\odot}$. The decrease in flux variability amplitude during eclipses suggests the presence of a nonvariable optical emission component with a magnitude $m_{R} \approx 13.2$.
\end{abstract}

Key words: massive binaries, microquasars, accretion disks, fast variability, optical observations

\section{INTRODUCTION}

The object SS 433 is a binary system with coninuous accretion onto a compact object, most probably a black hole (for a review see, e.g., Fabrika, 2004). It is the only known object of this kind in Galaxy and similar objects seen faceon are probably observed as ultraluminous X-ray sources in other galaxies (Fabrika \& Mescheryakov, 2001; King, 2002; Begelman et al., 2006).

The emission from SS 433 is variable at all observed time scales and at all wavelengths of the electromagnetic spectrum (see, e.g., Cherepashchuk, 1981; Gladyshev et al., 1983; Stewart et al., 1987; Goransky et al., 1998b; Eikenberry et al., 2001; Cherepashchuk et al., 2005; Kotani et al., 2006; Trushkin et al., 2007). Several types of periodic variability were detected in the variability of the source: precessional, orbital, and nutational; this allowed the parameters of the binary system to be constrained significantly (Cherepashchuk, 2002). The studies of eclipses also allowed to estimate the geometrical sizes of the various

\footnotetext{
*e-mail: rodion@hea.iki.rssi.ru
}

components of the binary system (see, e.g., Stewart et al., 1987).

The aperiodic variability of SS 433 was also studied (Revnivtsev et al., 2004, 2006). It was shown that the the broadband power spectrum of SS 433 is a power law with a break at a frequency of about $10^{-7} \mathrm{~Hz}$ below which the power spectrum is flat. Such a power spectrum is expected in the model of self-similar production of accretion-rate variability in an accretion disk, and the spectrum flatness at low frequencies is explained by the fact that no additional variability can be produced on time scales longer than the viscous time scale in accretion disk (Lvubarskii, 1997; Churazov et al., 2001; Gilfanov \& Arefiev, 2005).

Currently, the variability of SS 433 is poorly studied at higher frequencies $\gtrsim 10^{-3}-10^{-2} \mathrm{~Hz}$ (Fabrika, 2004; Revnivtsev et al., 2006). On the other hand, these frequencies should correspond to the size of X-ray and optical emission regions in the object and it should take its effect in the variability of the source at these high frequences. In this paper we present the results of our study of optical variability of SS 433 at high frequencies, using a significant amount of 
new data on fast optical photometry of SS 433 with $\approx 1 \mathrm{~s}$ time resolution.

\section{OBSERVATIONS}

The observations were carried out with Russian-Turkish 1.5-m telescope (RTT150) ${ }^{1}$, using CCD-photometer at the Cassegrain focus of the telescope $f=1 / 7.7$. Photometrical measurements were done with Andor DW-436 CCD camera. This is a $2048 \times 2048$ back-illuminated CCD, cooled electronically to $-60 \mathrm{~K}$. This $\mathrm{CCD}$ has high quantum efficiency (more then $90 \%$ in $R$ band), negligibly small dark current and low readout noise $(\approx 2 e)$. At the telescope focus the angular size of CCD pixel is $0.24^{\prime \prime}$, the size of field of view - about $8^{\prime}$.

\section{Instrument Setup}

In order to reduce the CCD readout time the observations were made as shown in Fig. 11. Since CCD readout speed depends mainly on linear size across the readout axis, telescope pointing was done so that the object and the reference star are set in parallel to that edge of the CCD, where the readout take place. The shutter was open during all the observations; therefore, both stars were set close to the readout side to avoid data contamination by bright stars outside the cutout strip.

Only the narrow CCD strip with the object and the reference star was read out during the observations. The strip width was chosen so that the main parts of the wings of the point spread function (PSF) were observed inside this strip. This strip did not change from observation to observation and its width was set to be $12^{\prime \prime}(50 \mathrm{CCD}$ pixels). The telescope was pointed so that these two stars were set at the center of the cutout CCD strip as precisely as possible; then the autoguider was immediately switched on. Since the telescope guiding accuracy is a few tenths of an arcsecond, one can be sure that both stars during the observations remained at the center of the cutout strip with good accuracy.

The CCD strip was read out not as an image but as a onedimensional data line by binning all 50 CCD pixels of the strip width into single data pixel (Fig. 1). In addition, the CCD was also binned by two pixels along the length of the cutout strip. This allowed to reduce significantly the readout time and readout noise, while the sky background was still much lower than the flux from the object. In this setup the readout time of our CCD was about $0.3 \mathrm{~s}$.

The exposure time was set to be about $0.7 \mathrm{~s}$ so that the exposure and readout cycle was exactly $0.974 \mathrm{~s}$ during all the observations. The data were initially written to computer random access memory and only every 1000 measurements were then written to the hard disk. This allowed to avoid the possible delays associated with the data writing to the hard disk within blocks of 1000 measurements.

\footnotetext{
${ }^{1}$ http://hea.iki.rssi.ru/rtt150/
}

The absolute timing marker was also placed in the data at the instant of their writing to the hard disk. Due to technical difficulties during our observations no accurate data timing based on the GPS signal was used. Instead, the computer clock was synchronized with the GPS signal every evening before and during the observations; the time markers obtained from this clock were then used. Thus, the absolute data timing accuracy should not be worse than a few tenths of a second.

It is important that our observing technique allow to obtain simultaneous measurements of the fluxes of at least two stars, the reference star and the object. This makes it possible to perform differential photometric measurements, which allows to take into account the extinction variations at different zenith distances and due to light clouds. In addition, as we show below, this also allows to take into account the most of the influence of the variable absorption and stellar jitter due to atmospheric turbulence up to frequencies of $\sim 0.5 \mathrm{~Hz}$.

The fluxes were measured inside the linear aperture centered on the profile of the signal from the star (Fig. 1). The window size was set for each segment of 1000 measurements separately at six RMS widths of the PSF. Thus, no more than a few tenths of a percent of the stellar flux was observed outside the aperture. The background was fitted by linear function in each strip being read out separately; during this procedure the stellar flux was eliminated by applying the standard sigma-clipping algorithm.

\section{The Influence of Atmospheric Turbulence on Photometric Measurements}

In order to study the aperiodic variability of various objects, the properties of the noise that emerges during the measurements of object fluxes must be well known. The true shape of spectrum of the object's variable emission will not be distorted if the flux measurement errors are independent in each measurement. In this case, the noise power spectrum is a constant that can be subtracted from the power spectrum of the measured emission of the object in order to obtain the power spectrum of its intrinsic variability.

The photon counting noise is definitely independent for different measurements, but in our case the errors could also emerge for other reasons. These primarily include the influence of atmospheric turbulence. There are always chaotic variations in the temperature and refractive index of the medium due to the presence of turbulence in the atmosphere. This leads to a distortion of the plane wave front, which manifests itself as jitter, a change of the shape, and scintillations of stars. All this, in turn, can affect the results of photometric measurements, with the corresponding measurement errors having a complex power spectrum dependent on the pattern of atmospheric turbulence.

According to the preliminary data of stellar jitter studies during RTT-150 observations, turbulence emerging on the telescope dome introduces a significant fraction of the dis- 

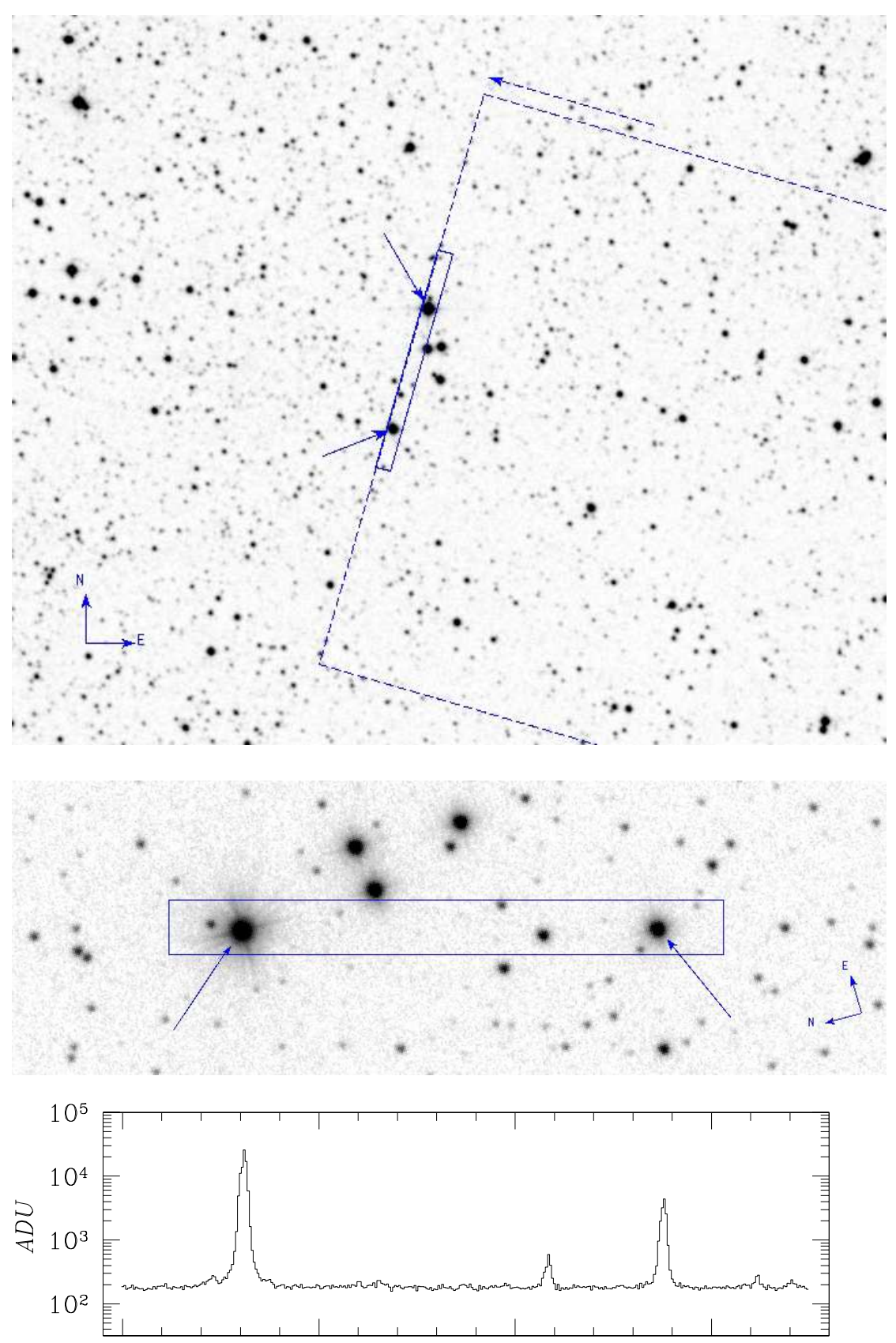

Fig. 1. The field near SS 433. We show here, how the telescope was pointed to object and what part of the CCD was used during our observations. The direction of CCD readout is shown with dashed arrow in the upper panel. The object and the reference star are also shown with arrows. In the lower panel the example of one-dimentional line of the data which was read out after the every exposure is shown. 


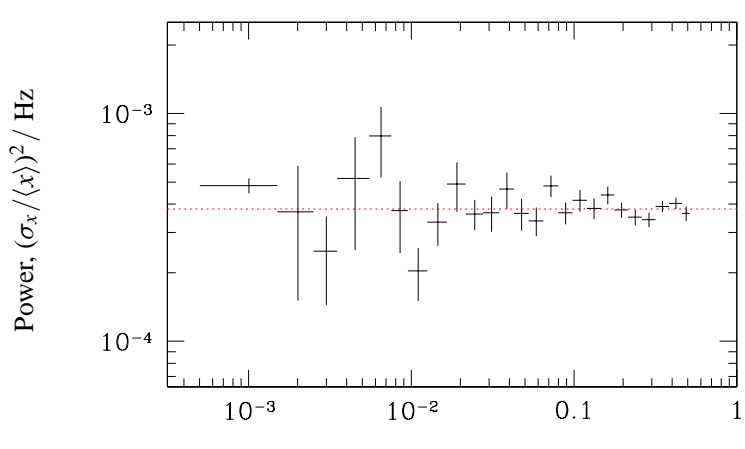

Frequency, $\mathrm{Hz}$

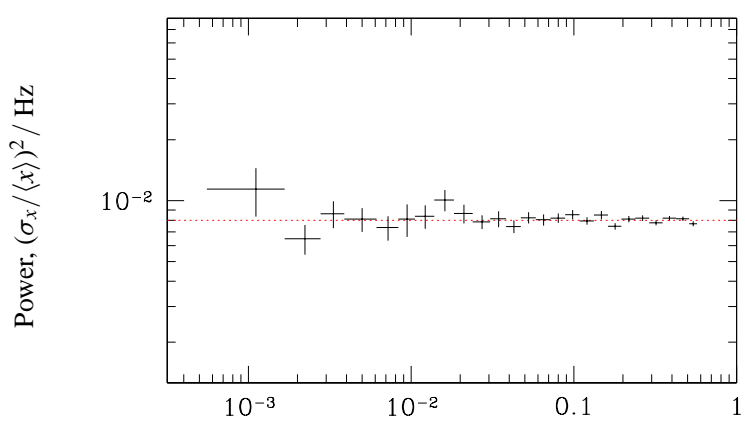

Frequency, Hz

Fig. 2. Power spectra of nonvariable stars. In the left panel - the results of the observations of nonvariable stars in the same nights when SS 433 was observed, in the right panel — the results of observations carried out later with slightly different signal-to-noise ratio.

tortions. This turbulence affects all stars in the telescope field of view in the very same way and, hence, it should not have any effect on differential photometric measurements.

Turbulence at high altitudes can also have a significant effect on the observations. Starting from the altitude of $2.5 \mathrm{~km}$, at which the TUBITAK Observatory is located, the bulk of the variability of the refractive index is gained up to an altitude of about $10 \mathrm{~km}$ (see, e.g., Zuev et al., 1988). At this altitude, the angular separation of $1.7^{\prime}$ between SS 433 and the reference star corresponds to a linear separation of about 3-4 m, which is larger than the telescope mirror size. Therefore, the light from these stars passes through different parts of the atmosphere and their jitter can differ. However, at a wind speed of $\sim 10 \mathrm{~m} / \mathrm{s}$, the turbulence will be essentially averaged out at a time scale of $\sim 1 \mathrm{~s}$ in a fixed region with a size of the order of the telescope mirror size. Therefore, one may expect that this turbulence will not contribute strongly to the photometric measurement errors as well.

In order to test this assumption directly, we carried out the observations of nonvariable stars with the same instrument setup that was used for the observations of SS 433 . The subsequent data reduction was also performed in exactly the same way. The derived power spectra are shown in Fig. 2. The left panel of Figure 2 shows the power spectrum of the nonvariable stars whose observations were carried out on the same nights as those of SS 433. The stars were chosen so that their fluxes were close to those from SS 433 and the reference star near this object. We see that, within the measurement errors, the shape of the power spectrum is consistent with the assumption that the power does not depend on frequency. In this case, the value of the constant power is close to what is obtained during the observations of SS 433 (see below).

The right panel of Figure 2 presents the results of our observations carried out on different nights and with slightly different CCD settings and stellar flux ratio. Within the errors, the shape of this power spectrum also agrees with a constant. During these observations, the signal-to-noise ra-

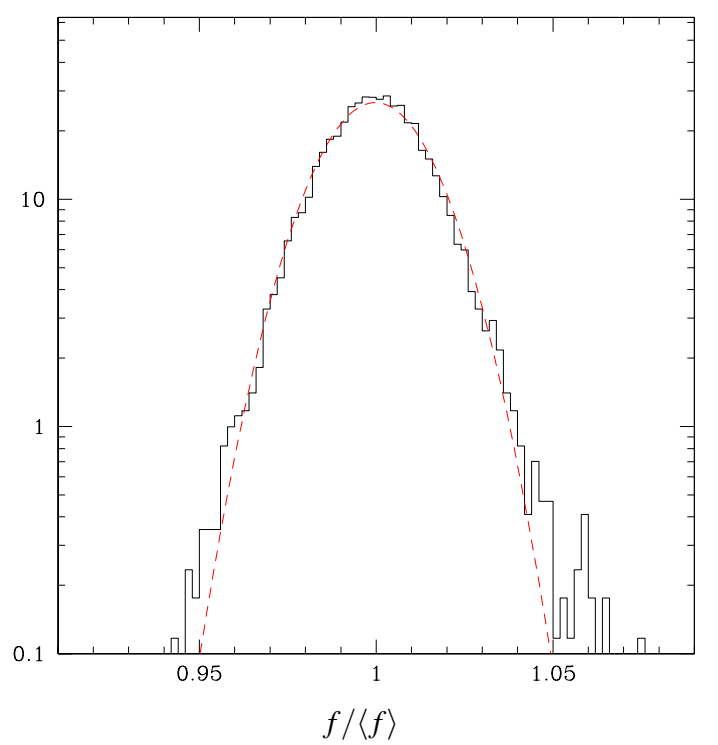

Fig. 3. The distribution of the errors in nonvariable stars flux measurements.

tio differ from that was obtained during the observations of SS 433. This is reflected in a different value of a constant in the observed power specrtum. However, all the uncertainties in the stellar fluxes measurements that can arise from atmospheric turbulence must be multiplicative (the variations in atmospheric transparency, the fraction of the flux in the PSF wings, and so on). Therefore, to estimate the possible distortions of the power spectrum, only the relative deviations from the constant value should be considered.

The distribution of errors in the flux measurements of nonvariable stars is shown in Fig. 3 In these observations, the stars were chosen so that the signal-to-noise ratio during these observations was close to that obtained in the observations of SS 433. One can see that, on the whole, this distribution is consistent with a Gaussian one; there are slight differences from the Gaussian distribution only at deviations 


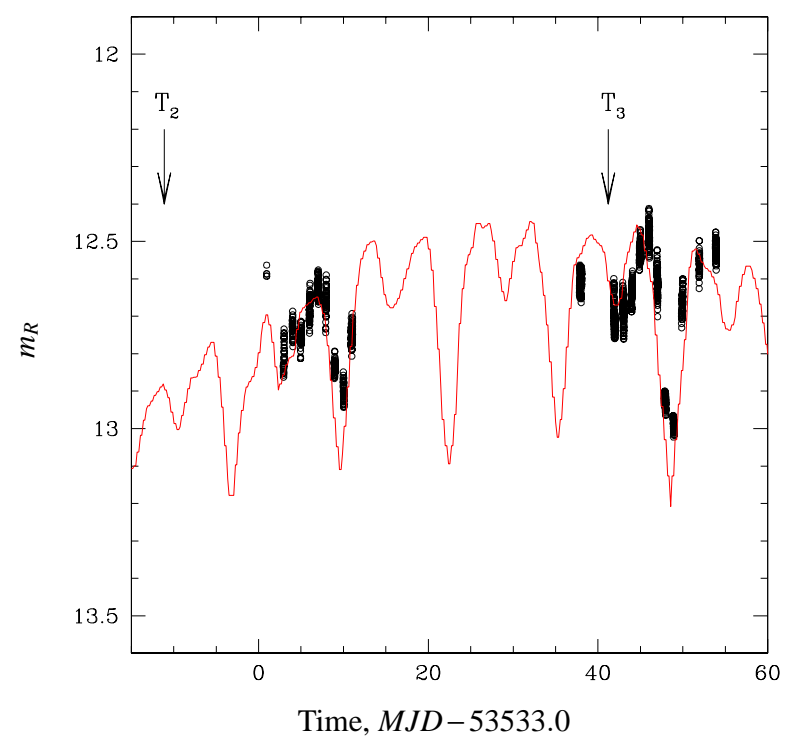

Fig. 4. The points shows the $R$-band magnitudes of SS 433 . Solid line shows the mean light curve in $V$ band from the observations of Goransky et al. (1998b), taken from the review by Fabrika (2004).

$>2 \sigma$.

Thus, the flux measurement errors in our observations of SS 433 are independent and Gaussian with a good accuracy. Therefore, the power spectrum of the object's intrinsic variability can be properly measured by subtracting the constant power which depends on the flux measurement errors during the observations.

\section{The Observations of SS 433}

The observations of SS 433 were carried out at RTT-150 telescope with CCD photometer, as was described above, mainly in the summer of 2005. In addition, a few observations were also carried out in the summer of 2004 and the fall of 2005. The observations were performed in the $\mathrm{R}$ band. The detailed data on these observations are presented in Table 1. The table provides the information on the date of observations (columns 1, 2, and 3), the time resolution during the observations ( $\Delta t$, column 4$)$, and the precession, orbital, and nutation phases of SS 433 at the time of observations (columns 5, 6, and 7, respectively), according to the ephemerides taken from the review by Fabrika (2004), see also Goransky et al. (1998b).

In Fig. 4 the $R$-band magnitude measurements for SS 433 obtained during our observations in the summer of 2005 are presented. The solid curve indicates the average $V$ band light curve constructed from the observations by Goransky et al. (1998b) taken from the review by Fabrika (2004). To make this curve coincident with our data, we set the phase based on the ephemerides of the orbital period taken from the same review and shifted the magnitudes so that they roughly coincided with our $R$-band observations. The arrows indicates the time of maximum of the pre-
Table 2. The results of our measurements

\begin{tabular}{ccc}
\hline \hline$M J D$ & $f$ & $\delta f$ \\
\hline 53247.959665 & 0.1324 & 0.0220 \\
53247.959670 & 0.1383 & 0.0229 \\
53247.959674 & 0.1242 & 0.0218 \\
53247.959679 & 0.1467 & 0.0214 \\
53247.959683 & 0.1296 & 0.0215 \\
53247.959688 & 0.1459 & 0.0218 \\
53247.959692 & 0.1220 & 0.0213 \\
53247.959697 & 0.1630 & 0.0239 \\
53247.959702 & 0.1588 & 0.0228 \\
53247.959706 & 0.1463 & 0.0230 \\
$\ldots$ & $\ldots$ & $\ldots$ \\
\hline
\end{tabular}

Note: - Here only a small part of the table is shown as an example. The whole table contains 238638 lines and is available in its entirety in the electronic version of the journal, and also at: http://hea.iki.rssi.ru/rtt150/en/ss433_pazh10/

cessional variability, when the accretion disk is maximally turned face-on to the observer's direction $\left(T_{3}, \varphi_{\text {prec }}=1\right)$, again according to the ephemerides from Fabrika (2004).

We see from Table 1 and Fig. 4 that the observations were carried out mainly close in time to the precession phase $T_{3}$. This was done to increase the sensitivity with respect to the variable emission of the object, because the variability amplitude is assumed to be highest at this precession phase. The observations were also performed during disk eclipses - a total of three eclipses were observed in the summer and the fall of 2005.

Due to weather restrictions and because of technical problems, the observations were not always carried out continuously during the night. Nevertheless, the bulk of the data are continuous time series of observations, each with a duration of several hours. In our work, we used a total of about 240 thousand optical flux measurements for SS 433; about 190 thousand are the measurements with a time resolution of about 1 s obtained during 2005. The results of our Rband flux measurements for SS 433 are summarized in Table 2 In Fig. 5 the examples of the measured SS 433 light curves with various time resolutions are shown. Here and everywhere below, unless stated otherwise, the optical flux from SS 433 is given in the R band in fractions of the flux of the reference star (see Fig. 1), whose magnitude, according to our measurements, is $m_{R}=10.57$.

The flux measurement errors differ from observation to observation, because they depend on the sky background, the PSF width determined by the degree of atmospheric turbulence, and the like. However, their value is about $2 \%$ of the measured flux in most observations. The observations performed in the late summer of 2004 are an exception, since the exposure times in these observations were reduced too much in order to achieve a higher time resolution.

The errors presented in Table 2 were determined from the Poissonian noise of the electrons recorded by the CCD. In 
Table 1. Observation Log

\begin{tabular}{|c|c|c|c|c|c|c|}
\hline \multirow{2}{*}{$\begin{array}{l}\text { Date } \\
\text { yymmdd }\end{array}$} & \multirow[t]{2}{*}{ Start } & \multirow[t]{2}{*}{ End } & \multirow{2}{*}{$\begin{array}{c}\Delta t, \\
\mathrm{~s}\end{array}$} & \multicolumn{3}{|c|}{ Phase, $\varphi$} \\
\hline & & & & prec. & orb. & nut. \\
\hline 040830 & 53247.9597 & 53247.9929 & 0.393 & 0.99 & 0.51 & 0.83 \\
\hline 040831 & 53248.8927 & 53248.9947 & 0.393 & 1.00 & 0.58 & 0.99 \\
\hline 040901 & 53249.8724 & 53249.9653 & 0.393 & 1.00 & 0.66 & 0.14 \\
\hline 050612 & 53533.9452 & 53533.9525 & 1.273 & 0.75 & 0.37 & 0.31 \\
\hline 050614 & 53535.9376 & 53536.0348 & 1.009 & 0.76 & 0.52 & 0.64 \\
\hline 050615 & 53536.9746 & 53537.0547 & 1.275 & 0.77 & 0.60 & 0.80 \\
\hline 050616 & 53537.9440 & 53538.0480 & 0.975 & 0.78 & 0.68 & 0.96 \\
\hline 050617 & 53538.9852 & 53539.0754 & 0.974 & 0.78 & 0.76 & 0.12 \\
\hline 050618 & 53539.9572 & 53540.0797 & 0.974 & 0.79 & 0.83 & 0.28 \\
\hline 050619 & 53540.9113 & 53540.9917 & 0.974 & 0.80 & 0.90 & 0.43 \\
\hline 050620 & 53541.9255 & 53542.0343 & 0.974 & 0.80 & 0.98 & 0.59 \\
\hline 050621 & 53542.9881 & 53543.0764 & 0.974 & 0.81 & 0.06 & 0.76 \\
\hline 050622 & 53543.8850 & 53544.0576 & 0.974 & 0.81 & 0.13 & 0.91 \\
\hline 050719 & 53570.8337 & 53571.0766 & 0.974 & 0.98 & 0.20 & 0.20 \\
\hline 050723 & 53574.8387 & 53575.0375 & 0.974 & 1.00 & 0.50 & 0.83 \\
\hline 050724 & 53575.8974 & 53576.0754 & 0.974 & 0.01 & 0.58 & 1.00 \\
\hline 050725 & 53576.9456 & 53577.0697 & 0.974 & 0.02 & 0.66 & 0.16 \\
\hline 050726 & 53577.8752 & 53578.0444 & 0.974 & 0.02 & 0.73 & 0.31 \\
\hline 050727 & 53578.9636 & 53579.0651 & 0.974 & 0.03 & 0.81 & 0.48 \\
\hline 050728 & 53579.9470 & 53580.0485 & 0.974 & 0.04 & 0.89 & 0.64 \\
\hline 050729 & 53580.8228 & 53580.9920 & 0.974 & 0.04 & 0.96 & 0.78 \\
\hline 050730 & 53581.8715 & 53581.9959 & 0.974 & 0.05 & 0.04 & 0.95 \\
\hline 050731 & 53582.8602 & 53582.9989 & 0.974 & 0.05 & 0.11 & 0.10 \\
\hline 050802 & 53584.9164 & 53584.9763 & 0.974 & 0.07 & 0.27 & 0.42 \\
\hline 050804 & 53586.8539 & 53586.9369 & 0.974 & 0.08 & 0.42 & 0.73 \\
\hline 051014 & 53657.8000 & 53657.8011 & 0.982 & 0.51 & 0.84 & 0.01 \\
\hline 051028 & 53671.7010 & 53671.7236 & 0.974 & 0.60 & 0.90 & 0.22 \\
\hline
\end{tabular}



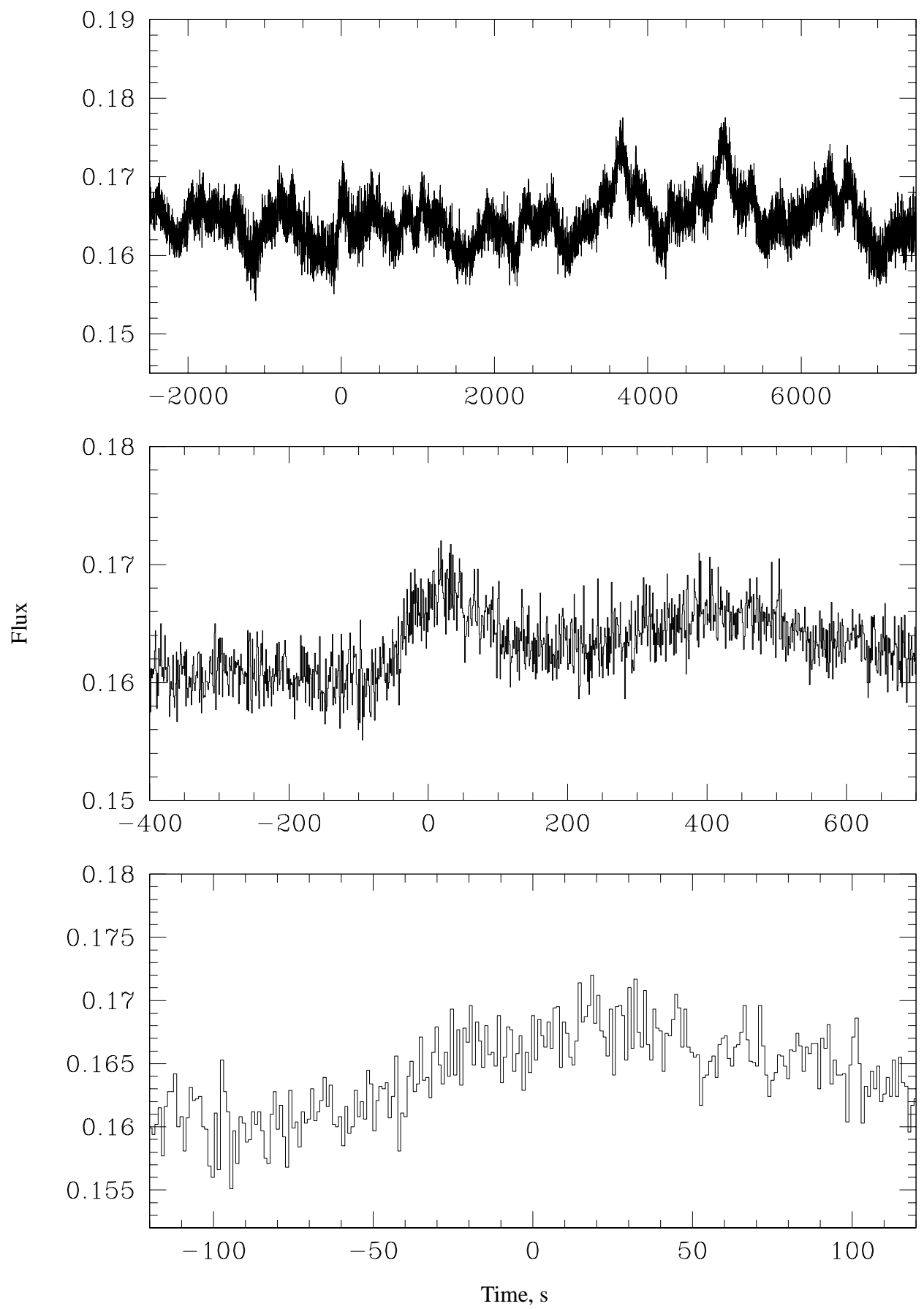

Fig. 5. A segment of the light curve of SS 433, shown at different time scales. The zero time on all panels corresponds to MJD=53577.92245. 


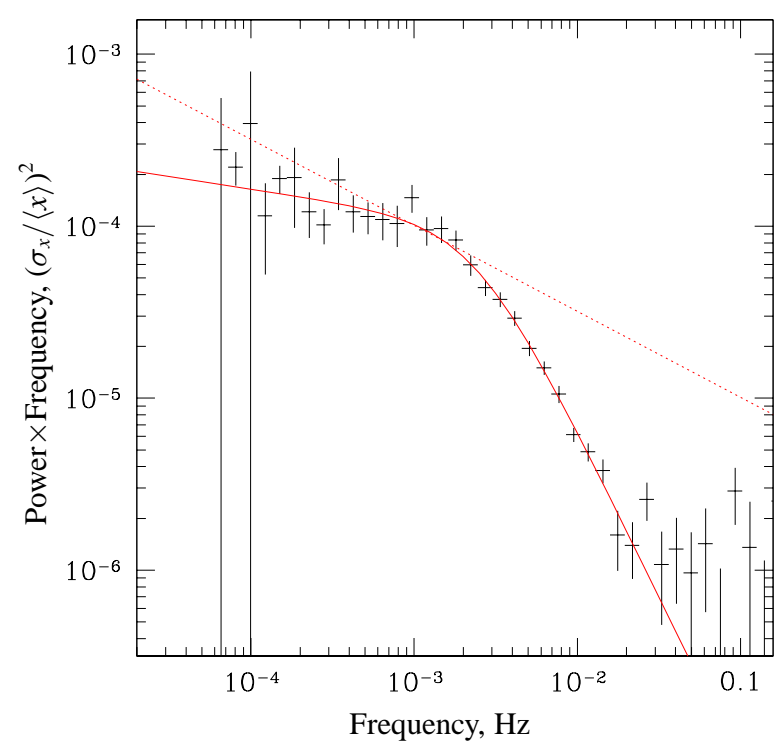

Fig. 6. Power specrtum of optical flux variability of SS 433 during the periods of no accretion disk eclipses.

order to take into account the influence of atmospheric jitter and other effects, this noise was multiplied by the correction factor calculated from the observations of nonvariable stars, which turned out to be equal to $\approx 2$. Thus, the errors in Table 2 must be close to the true measurement errors. They can be used to estimate the data quality. However, these errors are not used below to study the variability of the object.

\section{POWER SPECTRA}

The power spectra of the optical variability of SS 433 obtained from our light-curve measurements are shown in Figs 6 and 7 They were measured by averaging the Lomb-Scargle periodograms (Lomb, 1976; Scargle, 1982) that were calculated from all continuous data segments and renormalized so that to obtain the spectral power density of the variability in units of the fractional RMS squared. In Figs. 6 and 7 the spectral power density was additionally multiplied by the frequency in order to compare the power in the characteristic frequency intervals.

The constant corresponding to the Gaussian noise of the measurement errors is subtracted from these power spectra. This constant was determined from the power at frequencies $f>0.2 \mathrm{~Hz}$. Therefore, it was assumed that the entire variability at these frequencies is explained by the measurement errors. This should be close to reality with a good accuracy, because even at frequencies $f>0.03 \mathrm{~Hz}$ the power is observed to be almost exactly constant and its value, from $3 \times 10^{-4}$ to $4 \times 10^{-4}$ for different light-curve segments, agrees well with the constant power that was obtained during our observations of nonvariable stars with close magnitudes (see Fig.2).

Figure 6 shows the power spectrum of the variability of

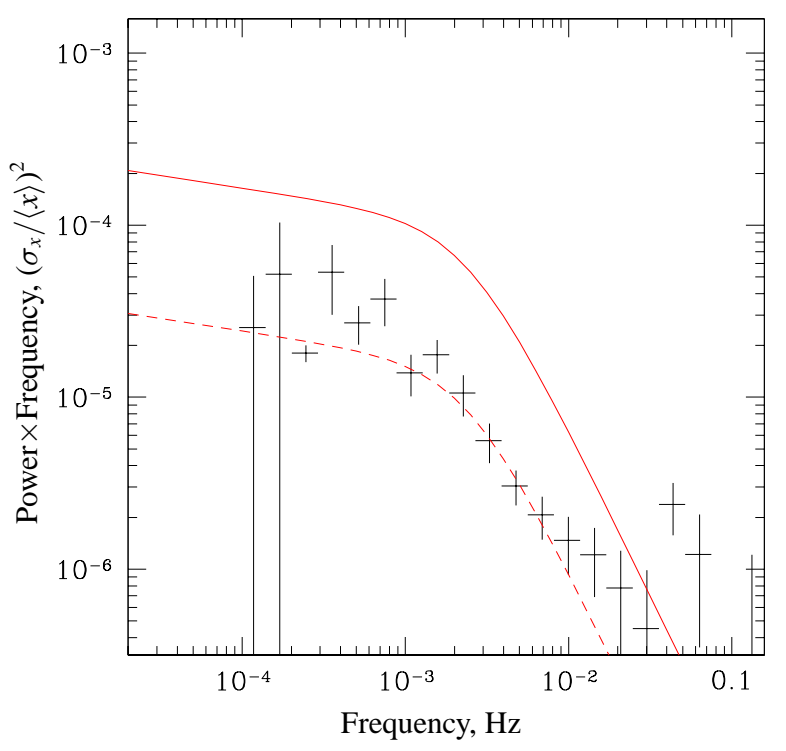

Fig. 7. Power specrtum of optical flux variability of SS 433 during the eclipses of the accretion disk.

that part of the light curve, where the accretion-disk eclipses were excluded, i.e., the light curve at the orbital phase $\left|\varphi_{\text {orb }}\right|>0.1$. The best fit model $P(f)=f^{\alpha_{1}}\left[1+\left(f / f_{0}\right)^{2 \alpha_{2}}\right]^{1 / 2}$, where $P(f)$ is the spectral power density, is also shown in the Figure. The best fit parameters for this model are $\alpha_{1}=-1.15 \pm 0.06, \alpha_{2}=-1.80 \pm 0.13$ and $f_{0}=2.43 \pm 0.29$. $10^{-3} \mathrm{~Hz}$. The dotted line in the Figure shows the power law with a slope of -1.5 that was obtained from the observations of SS 433 variability in the frequency range $10^{-7}-10^{-2} \mathrm{~Hz}$ (Revnivtsev et al., 2006).

The power-law slope of the power spectrum at high frequencies is $\alpha_{1}+\alpha_{2}=-2.95$, i.e., it is steeper as compared to the slope of the spectrum at low frequencies measured from our data $\left(\alpha_{1}=-1.15\right)$ and to the slope $\alpha_{1}=-1.5$, which was measured from the observations at lower frequencies by Revnivtsev et al. (2006). Thus, our data reveal a break in the power spectrum at a frequency of near $2.4 \cdot 10^{-3} \mathrm{~Hz}$.

In Fig. 7 the power spectrum from our observations during accretion-disk eclipses at an orbital phase $\left|\varphi_{\text {orb }}\right|<0.1$ is shown. We see that the flux variability is greatly reduced during disk eclipses. In this case, as far as can be judged from the data, the shape of the power spectrum remains approximately the same as that outside eclipses, while the normalization decreases approximately by a factor of 4.5. As an example, in Fig. 8 we show the segments of the light curves for SS 433 outside and during accretion-disk eclipses. The drop in the optical variability amplitude during the eclipse is seen in this Figure with the naked eye.

In Figs. 9 and 10 we show the relation between the mean optical flux from SS 433 and its RMS at $1000 \mathrm{~s}$ time scale. The filled circles in Fig. 10indicate the measurements when the orbital phase is $\left|\varphi_{\text {orb }}\right|<0.1$ (eclipse), while the open circles correspond to $\left|\varphi_{\text {orb }}\right|>0.1$ (out of eclipse). The mea- 


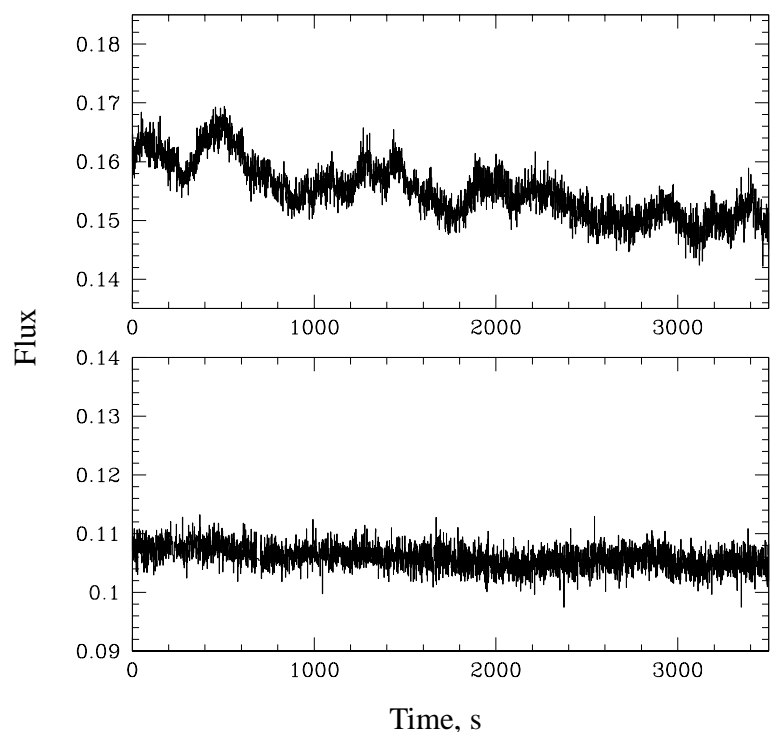

Fig. 8. The examples of SS 433 light curves. Upper panel - out of the eclipses of accretion disk, lower panel — during the eclipse.

surements with fluxes about 0.14 at the eclipse phase and with fluxes about 0.09 at the phase outside eclipse correspond to the observations on July 19 and September 28, 2005, respectively, when the orbital phase is close to a boundary value of $\varphi_{\text {orb }} \approx 0.9$. Thus, it follows from our data that the eclipse ingress occurs at slightly different times at different precession phases.

With the exception of these points, all of the remaining measurements show not only a drop in optical flux during eclipse but also a significant drop in the variability amplitude. In addition, we see from Figs. 9 and 10 that the optical variability amplitude decreases not only during the eclipses but also when the optical flux outside eclipse decreases. However, it turns out that this occurs slightly more slowly than during eclipse. In Fig. 10 the linear regressions for the data at fluxes greater and less than 0.14 are shown. We see that these lines have different slopes and the calculations of their slope estimates shows that they are different at a confidence level of about $3 \sigma$.

The change in the slope of the RMS - mean flux relation in these two cases is not something unexpected, because the accretion-disk eclipse by the companion star changes the ratio of the fractions of the fluxes from the variable and constant components in the total optical emission recorded on Earth. Indeed, a direct proportionality between the amplitude of aperiodic flux variations and the mean flux is commonly observed in accreting binary systems (see, e.g., Lyutyi \& Oknyanskii, 1987; Uttley \& McHardy, 2001). If we could observe only one variable emission component of the binary system (as it is observed, for example, in the Xray energy band), then one would expect the same direct proportionalyty between RMS and mean flux to be observed at all fluxes. However, the presence of a significant contri-

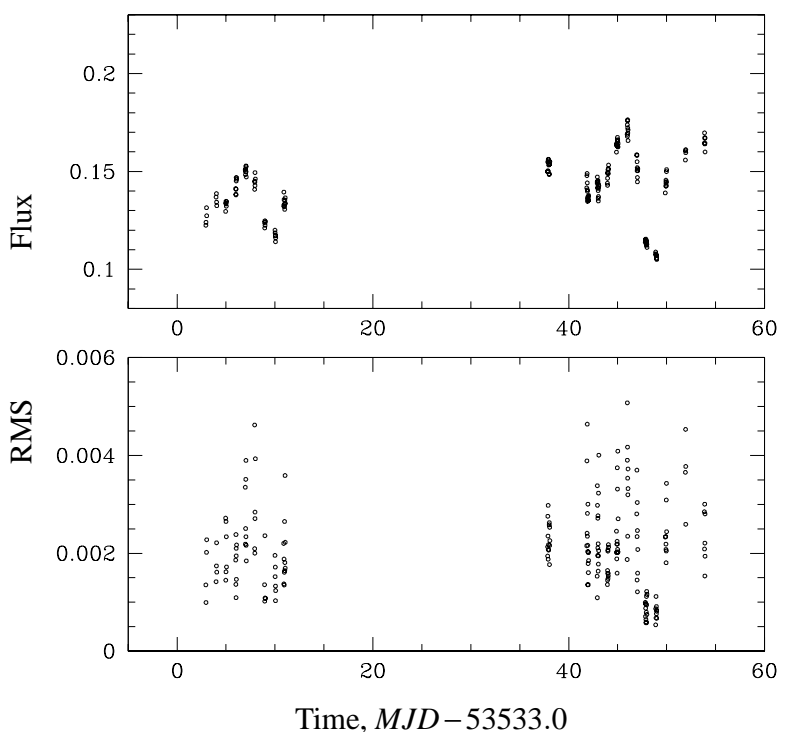

Fig. 9. SS 433 optical flux (upper panel) and its RMS at $1000 \mathrm{~s}$ time scale (lower panel).

bution of the nonvariable flux from the companion star in the optical band changes this relation. During the eclipse, the fractions of the variable and constant fluxes undergo additional changes, causing the slope of the RMS - mean flux relation to change relative to the out-of-eclipse case. We see from Fig. 10 that in the SS 433 optical emission during eclipses there is a nonvariable component with a flux of about 0.09 , which corresponds to a magnitude $m_{R} \approx 13.2$.

\section{VARIABILITY SMOOTHING AND GEOMETRY OF THE EMITTING REGION}

As it was shown above, in the power spectrum of the optical variability of SS 433 obtained from our measurements out of the accretion-disk eclipses there is a break at a frequency of about $2.4 \times 10^{-3} \mathrm{~Hz}$. The presence of a break in the variability power spectrum at high frequencies is expected, because the variability must disappear at small time scales comparable to the time delay of the photon arrival from different parts of the source of variable emission. A frequency $f \approx 2.4 \cdot 10^{-3} \mathrm{~Hz}$ corresponds to a characteristic time $T=1 / 2 \pi f \approx 70$ s and to a distance $R \approx 2 \cdot 10^{12} \mathrm{~cm}$.

The shape and exact position of the break in the power spectrum depends on the geometry of the source of variable emission. For example, if the entire variable emission originates on the surface of a homogeneous sphere of radius $R$ that, in turn, is illuminated by a central source, then the flux from an infinitely short flare of this source will be recorded by a remote observer as a flare with a time profile $\propto(1-t / R c)$. If the central source is intrinsically variable, then the flux recorded by a remote observer will correspond to the flux from the central source convolved with this response function, while the variability power spectrum 


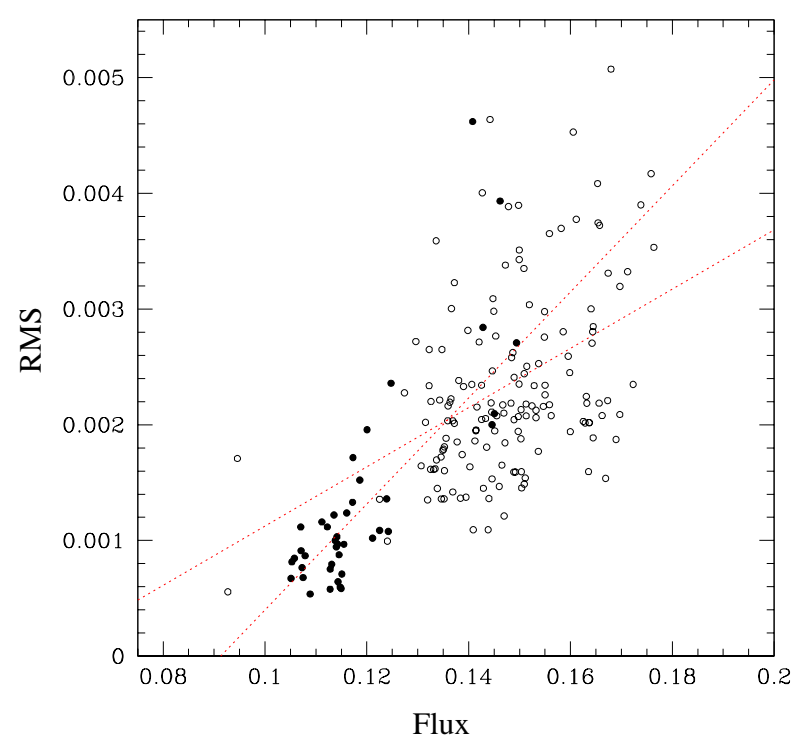

Fig. 10. RMS - optical flux relation for SS 433 at $1000 \mathrm{~s}$ time scale. The filled circles indicate the measurements during eclipse at $\varphi_{\text {orb }}<0.1$

of the flux recorded by the observer will be the product of the power spectrum of the intrinsic variability of central source and the square of the Fourier transform of the above response function.

To get an idea of how the variability power spectrum for such a sphere will appear, we performed the following simple simulation. At a $10^{4} \mathrm{~s}$ time interval (which roughly corresponds to the time of the continuous segment of observations in our data), we simulated a light curve with a power-law variability power spectrum $P \propto f^{-1.5}$, which was then convolved with the response function of the sphere discussed above. After that, the power spectrum of this time serie was obtained through the same procedure that was used to calculate the power spectra when reducing the observational data.

The power spectrum obtained in this way is shown in Fig. 11 with the filled strip. The solid curve indicates an analytical model fit of this power spectrum in the form $P(f)=f^{\alpha_{1}}\left[1+\left(f / f_{0}\right)^{2 \alpha_{2}}\right]^{1 / 2}$ with parameters $\alpha_{1}=-1.09$, $\alpha_{2}=-2.49, f_{0}=2.43 \cdot 10^{-3} \mathrm{~Hz}$. To construct this power spectrum, we took the sphere size to be $R=2.46 \cdot 10^{12} \mathrm{~cm}$ $(82.1 \mathrm{~s})$. This size was chosen so that the break occurred at a frequency $f \approx 2.43 \cdot 10^{-3} \mathrm{~Hz}$, as it is observed in our data on the variability of SS 433 . The slope of this power spectrum at high frequencies is found to be steeper than that observed for SS 433 (Fig. 6). This probably suggests that the emitting region actually has a more complex geometry.

In addition to the break at the characteristic frequency, the model power spectrum in Fig. 11]also exhibits other distortions related to the power spectrum measurement procedure - due to the red noise leakage and other effects, the power-law slope decreases at low frequencies and an additional small noise component is added at high frequencies,

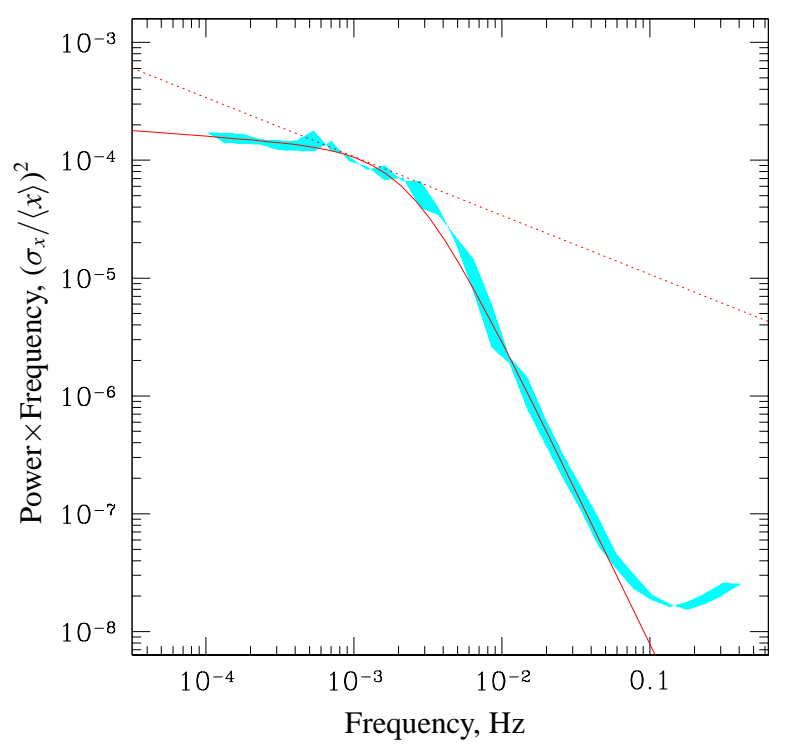

Fig. 11. The filled strip shows the power spectrum of the variability of the observed optical emission from a homogeneous sphere with a size $R=$ $2.46 \cdot 10^{12} \mathrm{~cm}(82.1 \mathrm{~s})$ whose surface emits variable radiation with a power spectrum $P \propto f^{-1.5}$ (dotted line). The solid curve indicates the model fit of this power spectrum.

up to the Kotelnikov-Nyquist frequency. The slope of the spectrum at frequencies near the lowest measured frequency decreases, because the observed power spectrum is a convolution of the true spectrum with the power spectrum of the window function and, hence, the power at the lowest observed frequencies is smeared toward higher frequencies.

Thus, the slope of the power spectrum at low frequencies is underestimated in our measurements. For the slope of the power spectrum at low frequencies to be properly measured, the corresponding simulations must be performed. We are going to do this in future. Nevertheless, these distortions should not affect strongly the measurement of the break frequency in the power spectrum.

From the above example we see that, given a specific model of the variable optical emission source in SS 433, from the observed power spectrum we can determine its geometrical size with a good accuracy and can even estimate the level of applicability of a particular geometrical model. For the most plausible models of the source, we are going to do this in future as well.

\section{DISCUSSION}

During our optical observations of SS 433, we used the CCD setup when not the image but only one-dimensional data line was read out after each exposure. This allowed to improve the time resolution of the photometric measurements to $\sim 1 \mathrm{~s}$. At the same time, the signal-to-noise ratio of optical flux measurements remains high - the scatter of the optical flux measurements for SS 433, whose magnitude is $m_{R} \approx 12.5$, constitutes only about two percent of the flux, 
i.e., it turns out to be comparable to the expected scatter due to Poissonian photon noise.

It is important that in these observations the possibility of simultaneous measurements of the fluxes from at least two stars (the object and the reference star) and obtaining diffrential flux measurements for the object is retained. Through the observations of nonvariable stars, we showed that this allows to remove almost completely the influence of atmospheric turbulence on the photometric measurements. Therefore, the data obtained by this technique are well suited for studying the aperiodic variability of various objects. We applied this observing technique recently in our study of the inner regions of the accretion disks in a sample of intermediate polars through the observations of their optical variability (Revnivtsev et al., 2010).

In this paper, we obtained and studied a large volume of data on the optical variability of SS 433. The observations were performed during more than twenty nights, mainly in the summer of 2005 . For our observations, we chose the precession phase when the accretion disk was maximally turned toward the observer, because the variability of the source at this phase is at maximum as well.

The power spectrum of the optical variability in SS 433 obtained from our light curve measurements out of accretion disk eclipses exhibits a break at a frequency of about $2.4 \cdot 10^{-3} \mathrm{~Hz}$. At higher frequencies the variability power falls down rapidly; the power-law slope of the power spectrum is $\approx-2.95$. In spite of this, a statistically significant optical variability of SS 433 is detected up to frequencies $\sim 3 \cdot 10^{-2} \mathrm{~Hz}$, with its relative amplitude at these frequencies being only of order $\sim 0.1 \%$.

We propose to explain the presence of a break in the variability power spectrum of SS 433 as a manifestation of the smoothing of the intrinsic variability of the source due to its finite size. Under this assumption our measurement of the break frequency gives a new, independent of other methods, measurement of the size of the variable optical emission source in SS 433. A frequency $f \approx 2.4 \cdot 10^{-3} \mathrm{~Hz}$ corresponds to a characteristic time $T=1 / 2 \pi f \approx 70 \mathrm{~s}$ and a size $R \approx 2 \cdot 10^{12} \mathrm{~cm}$. As we show above, a more accurate measurement of the size depends on the geometrical model of the source. However, given a specific model, we can determine its size from our data with good accuracy and can estimate the degree of the applicability of this partucilar geometrical model.

Our measurement of the size of the variable optical emission source turns out to be close to the estimate of the size of the hot optical emission source, $R \approx 2 \cdot 10^{12} \mathrm{~cm}$, obtained by modeling the energy distribution based on broadband photometry in the optical and ultraviolet wavelength bands (Dolan et al., 1997). A slightly smaller size, $R \approx$ $(0.06 \div 0.09) a \approx 3 \cdot 10^{11} \mathrm{~cm}$ is obtained from the measurement of the distance at which an X-ray emitting relativistic jet emerges from an opaque cloud of matter surrounding the source (Filippova et al., 2006). This may suggest that the optical hot variable source is asymmetric in projection onto the plane of the sky.

Our observations also show that the amplitude of the variability in SS 433 decreases sharply during accretion disk eclipses, but it does not disappear completely. This suggests that the size of the variable optical emission source is comparable to that of the normal star, which, consequently, should also be $R_{O} \approx 2 \cdot 10^{12} \mathrm{~cm} \approx 30 R_{\odot}$. The eclipse occurs as if, apart from the variable source, there is also a nonvariable source with a magnitude $m_{R} \approx 13.2$. This magnitude corrected for extinction $A_{V} \approx 8.4$ (Dolan et al., 1997) corresponds to the absolute magnitude $M_{R} \approx-7.2$. Note that about a half of the $R$-band emission originates in the wind around the binary system (Dolan et al., 1997; Goransky et al., 1998a).

Our estimates of the radius of the normal star and the absolute magnitude of nonvariable component in the source are consistent with the assumption that the normal star is an Atype supergiant, as it was suggested previously from photometric measurements during eclipses (Cherepashchuk et al., 1982) and from the observations of spectral lines of the normal star (Hillwig et al., 2004; Hillwig \& Gies, 2008). The radius of the normal star also agrees well with the estimate obtained from the observations of eclipses in X-rays (Filippova et al., 2006).

On the whole, the geometrical sizes of the source determined by studying the optical variability of SS 433 agree well with those measured previously by other methods. It should be emphasized that our measurements were obtained with a new, completely independent technique that uses a new physical phenomenon for such studies - the smoothing of the flux variability due to a finite light travel time across the size of the emitting system. Our results suggest that the existing views of the sizes of the system are mostly correct and contain no large systematic errors. In addition, we can conclude that the measurements of the aperiodic variability of X-ray binaries in the optical band is an efficient method of studying the geometry of the emitting regions in such systems.

We are grateful to TUBITAK National Observatory (TUG, Turkey), the Space Research Institute of the Russian Academy of Sciences (IKI RAN), and the Kazan State University for support in using the Russian-Turkish 1.5$\mathrm{m}$ telescope (RTT-150). This work was supported by the Russian Foundation for Basic Research (project nos. 0702-01004, 08-02-00974, 09-02-12384-ofi_m, 10-02-01442, 10-02-01145, 10-02-00492, 10-02-91223-ST_a), the Program for Support of Leading Scientific Schools of the Russian Federation (Nsh-5069.2010.2), and the Programs of the Russian Academy of Sciences P-19 and OPhN-16.

\section{REFERENCES}

Begelman M. C., King A. R., Pringle J. E., Mon. Not. R. Astron. Soc. 370, 399 (2006). 
Cherepashchuk A. ĐIJ., Mon. Not. R. Astron. Soc. 194, 761 (1981).

Cherepashchuk A. ĐIJ., Aslanov A. A., Kornilov V. G., Astron. Zh. 59, 1157 (1982).

Cherepashchuk A., Space Science Reviews 102, 23 (2002).

Cherepashchuk A. M., Sunyaev R. A., Fabrika S. N., Postnov K. A., Molkov S. V., Barsukova E. A., Antokhina E. A., Irsmambetova T. R., Panchenko I. E., Seifina E. V., Shakura N. I., Timokhin A. N., Bikmaev I. F., Sakhibullin N. A., Aslan Z., Khamitov I., Pramsky A. G., Sholukhova O., Gnedin Yu. N., Arkharov A. A., Larionov V. M., Astron. Astrophys. 437, 561 (2005).

Churazov E., Gilfanov M., Revnivtsev M., Mon. Not. R. Astron. Soc. 321, 759 (2001).

Dolan J. F., Boyd P. T., Fabrika S., et al., Astron. Astrophys. 327, 648 (1997).

Eikenberry S. S., Cameron P. B., Fierce B. W., Kull D. M., Dror D. H., Houck J. R., Margon, B., Astrophys. J. 561, 1027 (2001).

Fabrika S., Astrophysics and Space Physics Reviews 12, 1 (2004),; arXiv:astro-ph/0603390

Fabrika S., Mescheryakov A., "Galaxies and their Constituents at the Highest Angular Resolutions", Proceedings of IAU Symposium \#205, Ed. R. T. Schilizzi et al., Publisher Astronomical Society of the Pacific, San Francisco, California, p. 268. (2001).; arXiv:astro-ph/0103070

Filippova E., Revnivtsev M., Fabrika S., Postnov K., and Seifina E., Astron. Astrophys. 460, 125 (2006).

Gilfanov M., Arefiev V., arXiv:astro-ph/0501215.

Gladyshev S. A., Goransky V. P., Cherepaschuk A. M., Pis'ma v Astron. Zhurn. 9, 1 (1983) [Soviet Astron. Letters (Tr. Pisma Astr. Zh.), 9, 3].

Goransky V. P., Esipov V. F., Cherepaschuk A. M., Astron. Zh. 75, 209 (1998a) [Astronomy Reports, 42, 209].

Goransky V. P., Esipov V. F., Cherepaschuk A. M., Astron. Zh. 75, 383 (1998b) [Astronomy Reports, 42, 336].

Hillwig T. C., Gies D. R., Huang W., McSwain M. V., Stark M. A., van der Meer A., Kaper L., Astrophys. J. 615, 442 (2004).

Hillwig T. C., Gies D. R., Astrophys. J. 676, L37 (2008).

Kotani T., Trushkin S. A., Valiullin R., Kinugasa K., SafiHarb S., Kawai N., and Namiki M., Astrophys. J. 637, 486 (2006).

King A., Mon. Not. R. Astron. Soc. 335, L13 (2002).
Lomb N. R., Ap\&SS 39, 447 (1976).

Lyubarskii Yu. E., Mon. Not. R. Astron. Soc. 292, 679 (1997).

Lyutyj V. M., Oknyanskij V. L., Astron. Zh. 64, 465 (1987).

Revnivtsev M., Burenin R., Fabrika S., Postnov K., Bikmaev I., Pavlinsky M., Sunyaev R., Khamitov I., Aslan Z., Astron. Astrophys. 424, L5 (2004).

Revnivtsev M., Fabrika S., Abolmasov P., Postnov K., Bikmaev I., Burenin R., Pavlinsky M., Sunyaev R., Khamitov I., Sakhibullin N., Astron. Astrophys. 447, 545 (2006).

Revnivtsev M., Burenin R., Bikmaev I., Kniazev A., Buckley D. A. H., Pretorius M. L., Khamitov I., Ak T., Eker Z., Melnikov S., Crawford S., Pavlinsky M., Astron. Astrophys. 513, A63 (2010); arXiv:1002.4073.

Scargle J. D., Astrophys. J. 263, 835 (1982).

Stewart G. C., Watson M. G., Matsuoka M., Brinkmann W., Jugaku J., Takagishi K., Omodaka T., Kemp J. C., Kenson G. D., Kraus D. J., Mazeh T., Leibowitz E. M., Mon. Not. R. Astron. Soc. 228, 293 (1987).

Trushkin S. A., Nizhelskij N. A., Bursov N. N., Majorova E. K., "Black Holes from Stars to Galaxies - Across the Range of Masses”, Ed. V. Karas and G. Matt, Proceedings of IAU Symposium \#238, Cambridge, UK, Cambridge University Press, 463-464 (2007).

Uttley P., McHardy I. M., Mon. Not. R. Astron. Soc. 323, L26 (2001).

Zuev V. E., Banakh V. A., Pokrasov V. V., Contemporary ptoblems of athmospheric optics, vol. 5, "Optics of turbulent athmosphere”, Leningrad, Gidrometeoizdat, 1988 [in Russian]. 